Research paper

\title{
Target-induced natural killer cell loss as a measure of NK cell responses
}

\author{
Hilary S. Warren* \\ Cancer Immunology Research Unit, The Canberra Hospital, Australian Capital Territory, Australia \\ Department of Immunology, John Curtin School of Medical Research, Australian National University, Australian Capital Territory, Australia \\ Australian National University Medical School, Australian Capital Territory, Australia
}

\section{A R T I C L E I N F O}

\section{Article history:}

Received 17 March 2011

Received in revised form 2 May 2011

Accepted 1 June 2011

Available online 14 June 2011

\section{Keywords:}

Human NK cells

Cytotoxicity

CD107a

ADCC

Rituximab

\begin{abstract}
A B S T R A C T
The interaction of natural killer cells with susceptible target cells triggers NK cell activation, eliciting not only NK cell cytotoxicity and cytokine secretion, but also NK cell death. This study shows that following target cell interaction there is a substantial loss of NK cells, the extent of which correlates with measures of NK cell cytotoxicity assessed by the target cell release of ${ }^{51} \mathrm{Cr}$ and by the externalisation of the lysosomal marker LAMP-1 (CD107a) which is assessed on the remaining NK cells. This is the case for the killing of K562 (natural killing) and the CD20 mAb (Rituximab)-mediated killing of RAJI cells and autologous B cells (antibody-dependent cell cytotoxicity). This target-induced NK loss (TINKL) provides a sensitive and specific measure of NK cell responses appropriate to a clinical laboratory setting.
\end{abstract}

(c) 2011 Elsevier B.V. All rights reserved.

\section{Introduction}

Measuring NK cell activity is important in the overall assessment of patient immune function. The traditional cytotoxicity assay of ${ }^{51} \mathrm{Cr}$-release from labelled target cells (Brunner et al., 1968) is being superseded by flow based measures of NK cell cytotoxicity such as the target cellinduced externalisation of the lysosomal protein LAMP-1 (CD107a) (Alter et al., 2004; Fauriat et al., 2008). The response of NK cells to target cells is complex, since in addition to target cell killing, NK cells are stimulated to secrete cytokines and proliferate (reviewed in (Warren, 1996)), yet also undergo apoptosis (Jewett and Bonavida, 1996; Yamauchi et al., 1996).

In this paper data are presented showing that there is a substantial loss of NK cells induced as a consequence of interaction with sensitive target cells either through the process of natural killing or antibody-dependent cell cytotoxicity (ADCC). Quantifying this target-induced NK cell loss (TINKL) is shown to

\footnotetext{
* Cancer Immunology Research Unit, The Canberra Hospital, PO Box 11, Woden, ACT 2606, Australia. Tel.: +61 26244 2928; fax: +61 262443092

E-mail address: Hilary.Warren@anu.edu.au.
}

be a sensitive and specific measure of NK cell responses to target cells, and should prove a convenient assay for assessing NK cell responses in a clinical laboratory setting.

\section{Materials and methods}

\subsection{Antibodies and reagents}

CD3-FITC (BD Biosciences), CD3-PE (BioLegend) or CD3PerCP/Cy5 (BioLegend) was used to identify T cells. CD56-PE (Miltenyi Biotec), or CD56-FITC (BioLegend) with CD94-FITC (BioLegend), was used to identify NK cells. CD19-PE (BioLegend) was used to identify B cells. CD107a-PE (BioLegend) was used to identify LAMP-1 externalised on NK cells. Annexin-V-FITC (Calbiochem) was used to identify cells expressing cell surface phosphatidylserine, an early marker of apoptosis. Propidium iodide (BD Biosciences) was used to identify dead cells. The chimeric human/mouse anti-CD20 mAb Rituximab (Roche, Sydney, Australia) was obtained from the Pharmacy Department at the Canberra Hospital, as an unused portion from patient treatment. Human recombinant IL-2 (Millipore, Temecula, CA) was reconstituted according to the manufacturer's instructions. All reagents were tested for optimum concentrations. 


\subsection{Cell lines}

Target cells used were the NK sensitive K562 erythroleukaemic cell line, and the NK resistant B-lymphoblastoid cell RAJI. Cells were maintained by twice weekly subculture in RPMI-1640 containing $10 \%$ or $15 \%$ heat-inactivated foetal bovine serum (FBS) (TRACE Biosciences), respectively. The day prior to the assay, target cells were sub-cultured at $2 \times 10^{5} / \mathrm{ml}$ to ensure that they were in exponential growth phase for the assay. Typically target cells were propagated in $1 \mathrm{ml}$ volumes in 24 well plates (Linbro 0976063, MP Biomedicals).

\subsection{PBMC isolation}

Blood was obtained by informed consent under studies approved by the Human Ethics Committees of the ACT Department of Health and Community Care and the Australian National University, Canberra, Australia. Whole blood was collected in heparinised tubes. The blood was diluted with an equal volume of phosphate buffered saline (PBS), then under laid with Ficoll-Paque ${ }^{\mathrm{TM}}$ PLUS (GE Healthcare Bio-Sciences $A B$, Uppsala, Sweden) and centrifuged at $700 \mathrm{~g}$ for $30 \mathrm{~min}$. Peripheral blood mononuclear cells (PBMC) were harvested from the FicollPaque/plasma interface and washed 4 times with PBS until free of platelets ( $250 \mathrm{~g}$ for $10 \mathrm{~min}$ ). PBMC were frozen in liquid nitrogen using as cryopreservant, 10\% (v/v) CryoSure-DMSO (WakChemie Medical GMBH, Germany) prepared in Hanks balanced salt solution supplemented with 20\% FBS. PBMC were thawed rapidly and then diluted in 10 times the volume of pre-warmed PBS containing 20\% FBS. PBMC were washed twice with PBS/10\% FBS. Aliquots of fresh or thawed PBMC were cultured overnight in RPMI-1640 supplemented with 20\% FBS, $0.1 \mathrm{mM}$ 2-mercaptoethanol and $2 \mathrm{mM}$ L-glutamine, in a $5 \% \mathrm{CO}_{2}$ incubator. PBMC were cultured at a concentration of between $1 \times 10^{6}$ and $2 \times 10^{6}$ cells in $1 \mathrm{ml}$ volumes in 24 well plates.

\subsection{Cultures with target cells}

Unless otherwise stated cultures contained $5 \times 10^{4}$ PBMC with $5 \times 10^{3}$ target cells and were set up in triplicate in 96well round bottom plates (Costar 3799, Corning Inc., Corning $\mathrm{NY}$ ) in a final volume of $150 \mu \mathrm{lPMI}$ containing $10 \% \mathrm{FBS}$, with or without Rituximab. The optimum concentration of Rituximab in this assay was $1 / 10^{\text {th }}$ of the concentration giving maximum staining. Cultures were incubated at $37{ }^{\circ} \mathrm{C}$ in a $5 \%$ $\mathrm{CO}_{2}$ incubator for $6 \mathrm{~h}$ unless otherwise stated. At the end of the incubation period, the cultures were mixed using a multichannel pipettor and transferred to a 96-well V bottom plate (Linbro 76321, MP Biomedicals) for washing and staining with mAbs. In some experiments $25 \mu \mathrm{l}$ of a $1 / 5$ dilution of counting beads (Flow-Count ${ }^{\mathrm{TM}}$ Fluorospheres, Beckman Coulter) was pre-plated into the V wells. The cells were washed twice with PBS containing 5\% FBS and $0.1 \%$ sodium azide prior to staining with mAbs. T cells were identified using CD3-FITC, -PE or -PerCP/Cy5. NK cells were identified in the $\mathrm{CD}^{-}$gate using either CD56-PE or a combination of CD56-FITC and CD94-FITC. B cells were identified using a suboptimum concentration of CD19-PE in combination with an optimum concentration of CD3-PE to obtain a clear separation in the FL-2 channel. CD107a expression on NK cells was assessed using CD107a-PE added at the end of the $6 \mathrm{~h}$ culture (Fauriat et al., 2008). In all cases mAbs were prepared in PBS containing 5\% FBS and $0.1 \%$ sodium azide, and staining was for $30 \mathrm{~min}$ on ice followed by washing twice. Cell pellets were resuspended in $50 \mu \mathrm{PBS} / \mathrm{FBS} /$ azide and were fixed by adding an equal volume of $0.2 \%$ paraformaldehyde prepared in PBS. For analysis of Annexin-V binding, cells were washed in binding buffer (150 mM NaCl, $10 \mathrm{mM}$ Hepes pH 7.4, $5 \mathrm{mM} \mathrm{KCl,} 1 \mathrm{mM}$ $\mathrm{MgCl}_{2}, 1.8 \mathrm{mM} \mathrm{CaCl}_{2}$ ) (Purdue University Cytometry Laboratories, http://www.cyto.purdue.edu/flowcyt/research/ cytotech/apopto/data/chap16.htm) containing 0.1\% BSA immediately following $\mathrm{mAb}$ incubation. Annexin-V-FITC prepared in the binding buffer/BSA was added and the cells incubated for $10 \mathrm{~min}$ at RT and analysed immediately without fixation. To identify dead cells, propidium iodide $(1 \mu \mathrm{g} / \mathrm{ml})$ was added directly following mAb staining and cells were analysed immediately without fixation. Cells were collected on a FACScan (BD Biosciences) flow cytometer using linear Forward Scatter (FSC) and logarithmic Side Scatter (SSC) parameters.

\subsection{Data analysis}

Data were analysed using WinMDI 2.8 software (Purdue University) and FlowJo 8.8.7 software (Tree Star, Inc). Counting beads and live lymphocytes were gated using FSC/ SSC parameters. NK cells, T cells and B cells were gated based on the mAbs and fluorochromes used. Beads or T cells were used as references to evaluate NK cell loss in the cultures. The ratio of NK/T or NK/beads was determined for each culture, and NK loss as a consequence of target cell interaction was expressed as a percentage of the ratio in PBMC cultured without target cells. B cell loss in PBMC cultures was determined by comparing the $\mathrm{B} / \mathrm{T}$ ratio in cultures containing Rituximab compared to PBMC cultured in medium alone.

Target-induced expression of CD107a on NK cells in PBMC was recorded as percentage positive after subtraction of the background expression of CD107a on NK cells in PBMC cultured alone. Background expression of CD107a was set at between $1 \%$ and $2 \%$ positive. Similarly, the percentage of Annexin-V binding NK cells in PBMC cultured with target cells was determined following subtraction of any background binding of Annexin-V on NK cells in PBMC cultured alone.

\section{6. ${ }^{51} \mathrm{Cr}$-release assay}

Target cells were labelled with $\mathrm{Na}_{2}{ }^{51} \mathrm{CrO}_{4}(25 \mu \mathrm{Ci}$ per $5 \times 10^{5}$ cells per $100 \mu \mathrm{l}$ ) for $1 \mathrm{~h}$ at $37^{\circ} \mathrm{C}$ and washed 3 times. Cultures of PBMC and target cells were set up as described in Section 2.4 except that the culture period was 4 h. Following centrifugation, a $75 \mu \mathrm{l}$ aliquot of supernatant was harvested from the cultures and counted in a Packard Cobra 5000 gamma-counter. Target cells incubated alone gave background release, and CPM in the supernatant from these cultures was subtracted from CPM in the supernatant of PBMC/target cell cultures. Maximum release of ${ }^{51} \mathrm{Cr}$ from target cells was determined in triplicate using $50 \mu$ of target cells incubated with $450 \mu$ of $0.1 \%$ Triton X-100 for the $4 \mathrm{~h}$ culture period, and after centrifugation $250 \mu$ aliquots were counted. CPM in the supernatant of PBMC/target cell cultures was compared to the 

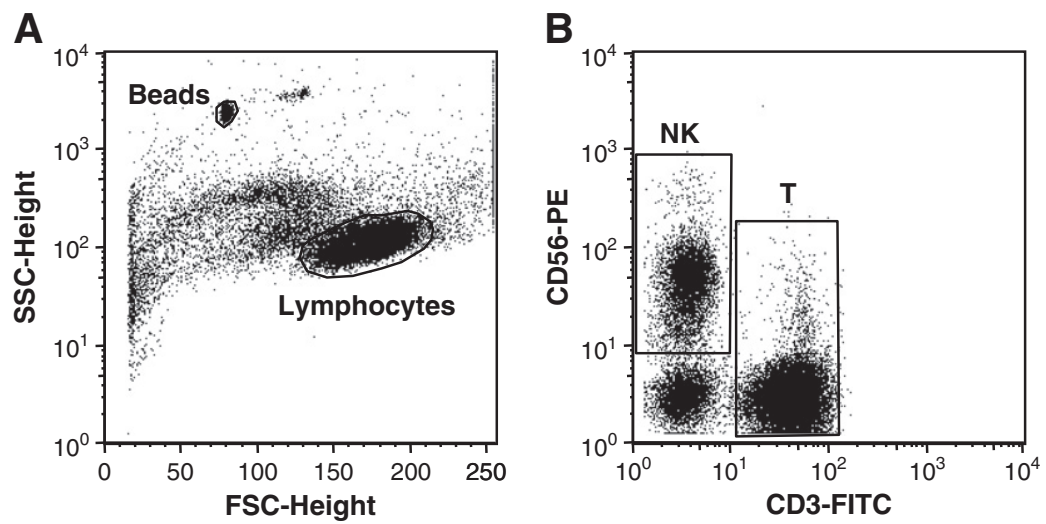

Fig. 1. Analysis of PBMC showing gating of lymphocytes and counting beads (A) and of NK cells and T cells within the lymphocyte gate (B).

maximum CPM released from target cells by Triton X-100. Cytotoxicity is expressed as percent specific lysis.

\section{Results}

\subsection{NK cell loss is a consequence of target cell interaction}

When PBMC are cultured with K562 (natural killing) or with the NK resistant target cell RAJI in the presence of the CD20 mAb Rituximab (ADCC), there is a loss of NK cells compared to PBMC cultured alone. Data from a typical experiment are shown in Fig. 1 and Table 1. Fig. 1A shows the FSC/SSC profile of PBMC and the gating of lymphocytes and counting beads. Separate experiments showed that the lymphocyte gate contained only viable cells as assessed by propidium iodide staining. Fig. 1B shows the gating of NK cells and T cells within the lymphocyte population. Table 1 presents data illustrating the method of quantifying NK cells in PBMC cultures. The ratio of NK/bead or NK/T cells in PBMC cultured with target cells is compared to the ratio in PBMC cultured alone, with the percentage of NK loss in target cell cultures recorded. Quantifying NK cells using either the $\mathrm{NK} /$ bead ratio or the NK/T ratio gave equivalent results. The results show that there is a substantial loss of NK cells as a consequence of natural killing and ADCC. Equivalent target-induced NK loss was observed using freshly isolated PBMC or PBMC obtained from liquid nitrogen storage. In other experiments, pre-incubation of PBMC with rIL-2 for up to $68 \mathrm{~h}$ prior to culturing with target cells neither enhanced nor diminished targetinduced NK loss (data not shown).

Data presented in Fig. 2 are from two donors representative of three different experiments that explore the effect of different culture parameters on target-induced NK cell loss. Fig. 2A shows that K562 targets induce progressive loss of NK cells across an $8 \mathrm{~h}$ culture period, and that NK cell loss as a consequence of ADCC is essentially unchanged over this time period. Fig. 2B shows that NK cell loss is consistent over a 6 -fold range of effector to target cell ratios.

Annexin- $\mathrm{V}$ binding, considered an early indicator of apoptosis, was assessed on NK cells in the lymphocyte gate following culture with target cells. The data in Fig. 3 are from

Table 1

Target cell-induced loss of NK cells.

\begin{tabular}{|c|c|c|c|c|c|c|c|c|c|}
\hline \multirow[t]{2}{*}{ Culture $^{\mathrm{a}}$} & \multirow[t]{2}{*}{ NK cells ${ }^{b}$} & \multirow[t]{2}{*}{ Beads } & \multirow[t]{2}{*}{$\mathrm{T}$ cells ${ }^{\mathrm{b}}$} & \multicolumn{2}{|c|}{$\begin{array}{l}\text { Ratio } \\
\text { NK/beads }\end{array}$} & \multirow[t]{2}{*}{$\%$ loss NK cells } & \multicolumn{2}{|l|}{$\begin{array}{l}\text { Ratio } \\
\text { NK/T }\end{array}$} & \multirow[t]{2}{*}{$\%$ loss NK cells } \\
\hline & & & & & Mean $\pm S D$ & & & Mean $\pm S D$ & \\
\hline \multirow[t]{3}{*}{ Medium } & 5312 & 2494 & 21588 & 2.130 & $2.250 \pm 0.179$ & Reference & 0.246 & $0.248 \pm 0.003$ & Reference \\
\hline & 5250 & 2426 & 21287 & 2.164 & & & 0.247 & & \\
\hline & 5164 & 2103 & 20504 & 2.456 & & & 0.252 & & \\
\hline \multirow[t]{3}{*}{ K562 } & 3550 & 2366 & 19763 & 1.500 & $1.623 \pm 0.121$ & $27.9 \pm 5.4$ & 0.180 & $0.183 \pm 0.004$ & $26.3 \pm 1.5$ \\
\hline & 3706 & 2277 & 20331 & 1.628 & & & 0.182 & & \\
\hline & 3937 & 2260 & 21069 & 1.742 & & & 0.187 & & \\
\hline \multirow[t]{3}{*}{$\mathrm{RAJI}+\mathrm{CD} 20 \mathrm{mAb}$} & 2342 & 2118 & 19906 & 1.106 & $1.127 \pm 0.019$ & $49.9 \pm 0.9$ & 0.118 & $0.124 \pm 0.005$ & $50.2 \pm 2.2$ \\
\hline & 2449 & 2139 & 19094 & 1.145 & & & 0.128 & & \\
\hline & 2600 & 2298 & 20850 & 1.131 & & & 0.125 & & \\
\hline
\end{tabular}

a PBMC were cultured for $6 \mathrm{~h}$ with medium, with K562 (natural killing) or with RAJI in the presence of the CD20 mAb Rituximab (ADCC).

${ }^{\mathrm{b}} \mathrm{NK}$ cells were defined as $\mathrm{CD} 3^{-} \mathrm{CD}_{56}{ }^{+}$, T cells were defined as $\mathrm{CD} 3^{+}$. 

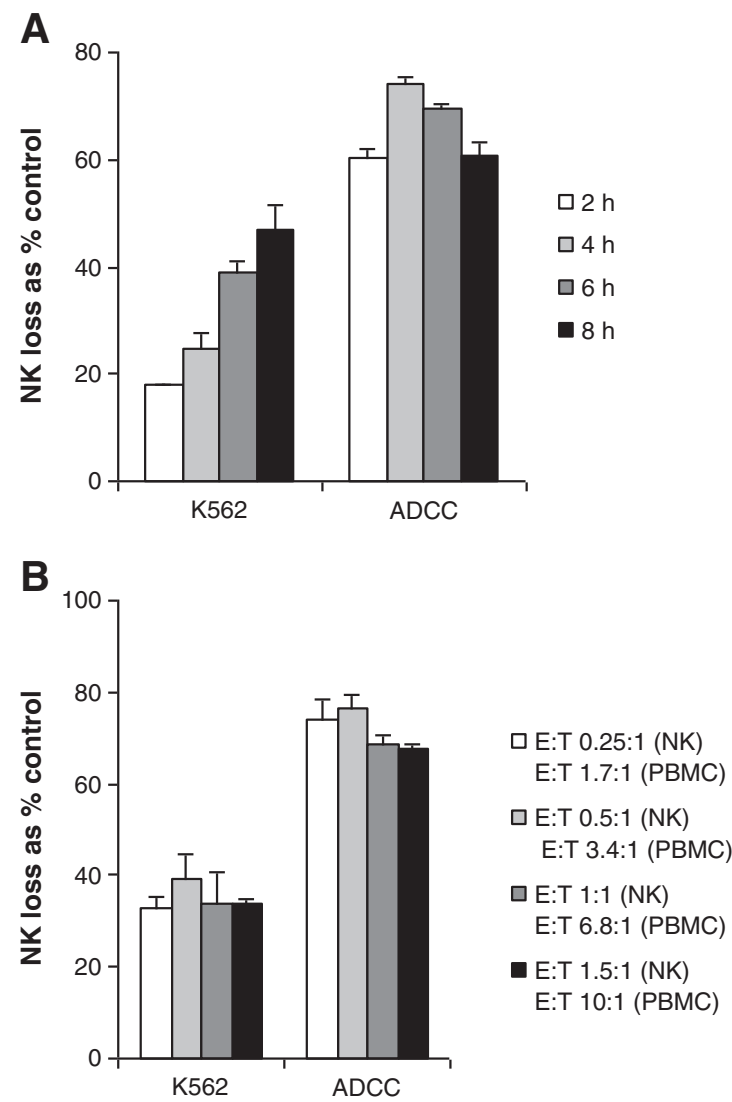

Fig. 2. Triplicate cultures were set up containing $5 \times 10^{4}$ PBMC (A) or varying numbers of PBMC (B), with either $5 \times 10^{3} \mathrm{~K} 562$ or $5 \times 10^{3}$ RAJI in the presence of Rituximab (ADCC). After varying times (A) or after $6 \mathrm{~h}$ (B) the cultures were analysed by immunofluorescence staining and flow cytometry. NK cell loss in cultures with target cells was compared to PBMC cultured alone using T cells as an internal control. The effector to target ratio is expressed in terms of PBMC and as actual NK cells present (B).

one experiment representative of several experiments with different donors showing that in addition to NK loss, a proportion of NK cells remaining in the lymphocyte gate gain

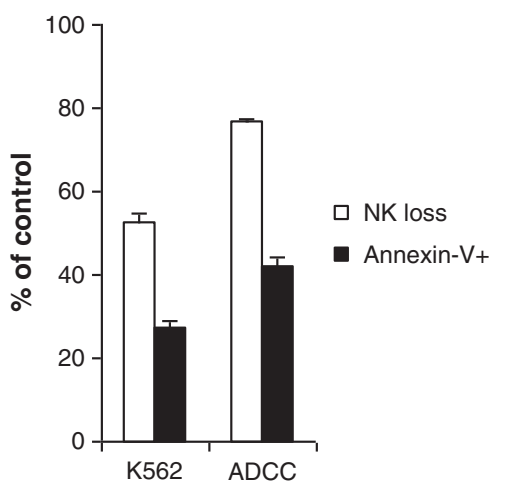

Fig. 3. Triplicate cultures were set up containing $5 \times 10^{4} \mathrm{PBMC}$ with either $5 \times 10^{3}$ K562 or $5 \times 10^{3} \mathrm{RAJI}$ in the presence of Rituximab (ADCC). At $6 \mathrm{~h}$ cultures were analysed by immunofluorescence staining and flow cytometry. NK cell loss in cultures with target cells was compared to PBMC cultured alone using T cells as an internal control. Annexin- $\mathrm{V}$ binding followed the $\mathrm{mAb}$ staining for NK cells and T cells. the ability to bind Annexin-V. Annexin- ${ }^{+}$NK cells were detectable as early as $2 \mathrm{~h}$ (data not shown). The proportion of NK cells in the lymphocyte gate binding Annexin-V paralleled

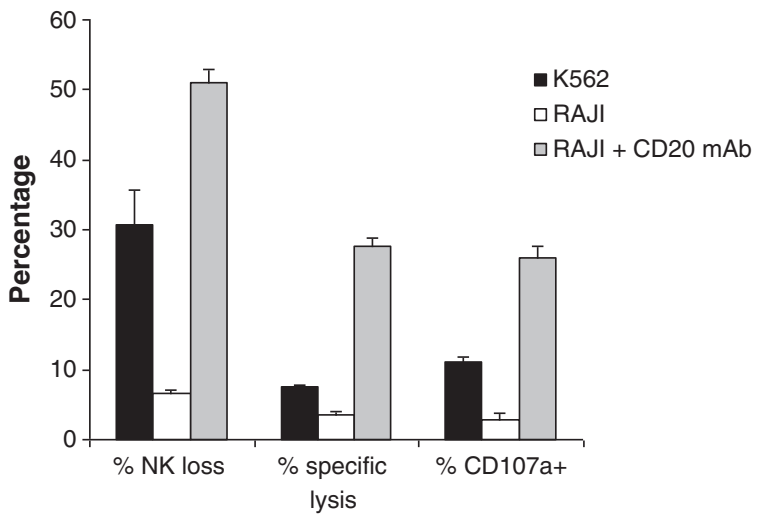

Fig. 4. For each assay, triplicate cultures were set up containing $5 \times 10^{4}$ PBMC (control), with either $5 \times 10^{3} \mathrm{~K} 562$ or with $5 \times 10^{3}$ RAJI in the absence or presence of Rituximab (ADCC). Cytotoxicity was assessed using ${ }^{51} \mathrm{Cr}$-labelled target cells in a $4 \mathrm{~h}$ assay. Expression of CD107a on NK cells and measurement of NK cell loss was determined after 6 h culture by immunofluorescence staining and flow cytometry. 

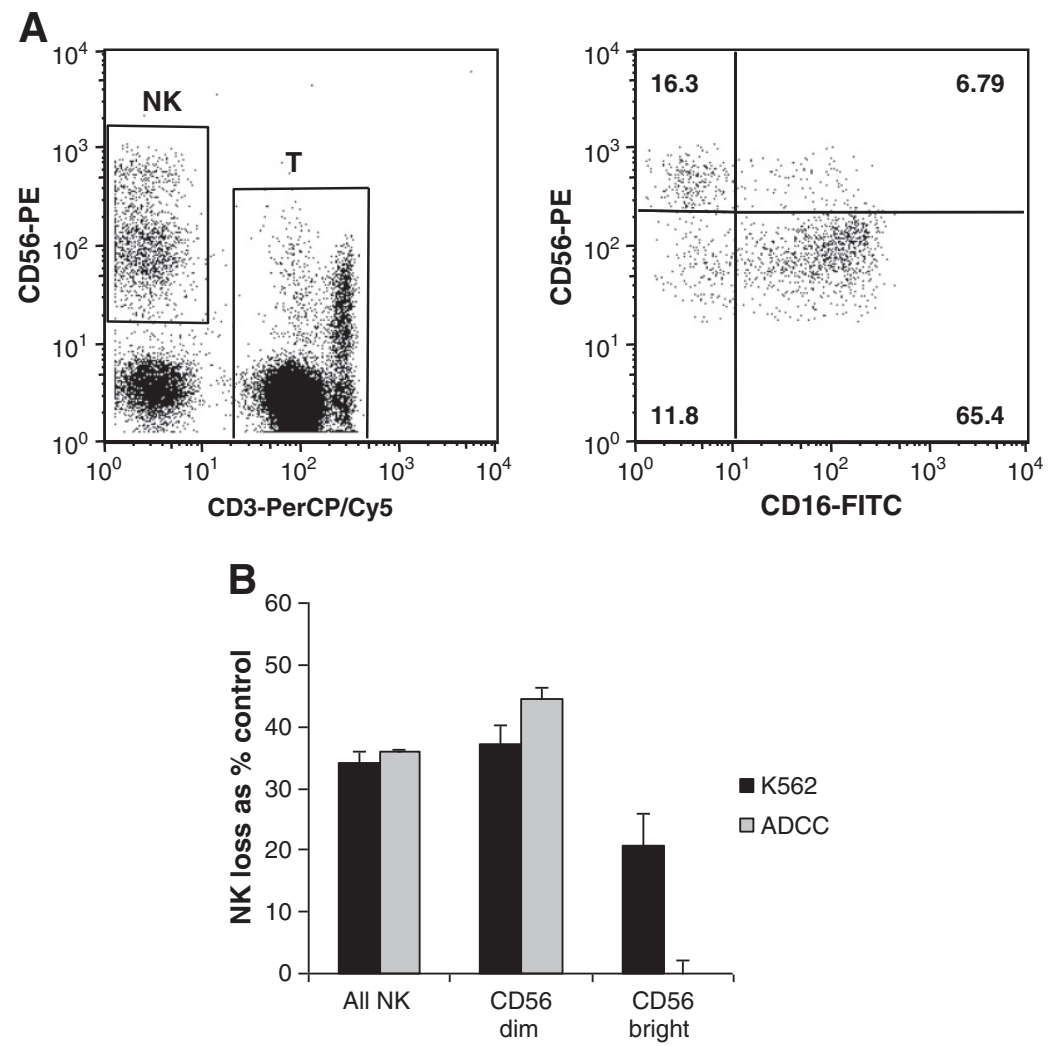

Fig. 5. Triplicate cultures were set up containing $5 \times 10^{4} \mathrm{PBMC}$ (control), with either $5 \times 10^{3} \mathrm{~K} 562$ or $5 \times 10^{3} \mathrm{RAJI}$ in the presence of Rituximab (ADCC). After $6 \mathrm{~h}$ cultures were analysed by immunofluorescence staining and flow cytometry. NK cells were defined as CD3 ${ }^{-} \mathrm{CD}_{5} 6^{+}$and then the CD56 $6^{\text {bright }}$ and CD56 ${ }^{\text {dim }}$ subsets were assessed (A). For this donor $7 \%$ of PBMC were NK cells, and of these $23.1 \%$ were CD56 bright the majority of which were CD16 ${ }^{-}$. NK cells were quantified relative to T cells as an internal control (B). The $100 \%$ control values corresponded to NK/T ratios of $0.08832 \pm 0.001$ (all NK), $0.07242 \pm 0.00164$ (CD56 ${ }^{\text {dim }}$ ) and $0.01589 \pm 0.00089$ (CD56 $\left.6^{\text {bright }}\right)$.

the proportion of NK cells lost from the lymphocyte gate following culture with target cells.

\subsection{Target-induced NK loss correlates with cytotoxicity} measured as target cell lysis and with the induced expression of CD107a on remaining viable NK cells

The data in Fig. 4 compares target-induced NK loss with target cell lysis measured by the ${ }^{51} \mathrm{Cr}$-release assay, and with target-induced expression of CD107a on the surface of the remaining viable NK cells. The data are representative of several experiments using PBMC from different donors. The results show that the loss of NK cells in cultures containing K562 or in cultures containing the NK resistant target RAJI in combination with the CD20 mAb Rituximab paralleled the specific lysis of target cells assessed by the ${ }^{51} \mathrm{Cr}$-release assay. The loss of NK cells in these cultures also paralleled the expression of CD107a which is measured on the remaining NK cells within the lymphocyte gate. There was minimal loss of NK cells in cultures containing the NK resistant RAJI cells in the absence of Rituximab. Of note is that measurement of NK cell loss is the most sensitive of the three assays.

\subsection{Target-induced NK loss is specific}

NK cells can be subdivided on the basis of their expression of CD56, with the majority of NK cells in PBMC being CD56 ${ }^{\text {dim }}$ and the minority being CD56 bright. These NK subsets have functionally distinct properties, as reviewed by Cooper and colleagues (Cooper et al., 2001). The majority of CD56 ${ }^{\text {dim }}$ NK cells express CD16 the required receptor for ADCC, whereas the majority of CD56 ${ }^{\text {bright }}$ NK cells do not. These subsets are illustrated in the example in Fig. 5A. Both NK cell subsets mediate natural killing, although the cytolytic activity of the CD56 ${ }^{\text {bright }}$ subset is weaker (Cooper et al., 2001). The results in Fig. 5B show the loss of NK cells following culture with K562 (natural killing) or RAJI and Rituximab (ADCC). As expected there is loss of CD56 ${ }^{\mathrm{dim}}$ NK cells when cultured with K562 or with RAJI and Rituximab. Specificity of NK loss is shown by the fact that there is no loss of CD56 ${ }^{\text {bright }} \mathrm{NK}$ cells when cultured with RAJI and Rituximab, yet there is loss of this subset when cultured with K562. This result was obtained with PBMC from several other donors. The extent of loss of CD56 ${ }^{\text {bright }} \mathrm{NK}$ cells is less than the loss of CD56 dim NK cells in cultures with K562, consistent with the notion that CD56 ${ }^{\text {bright }}$ NK cells are less cytolytic. 

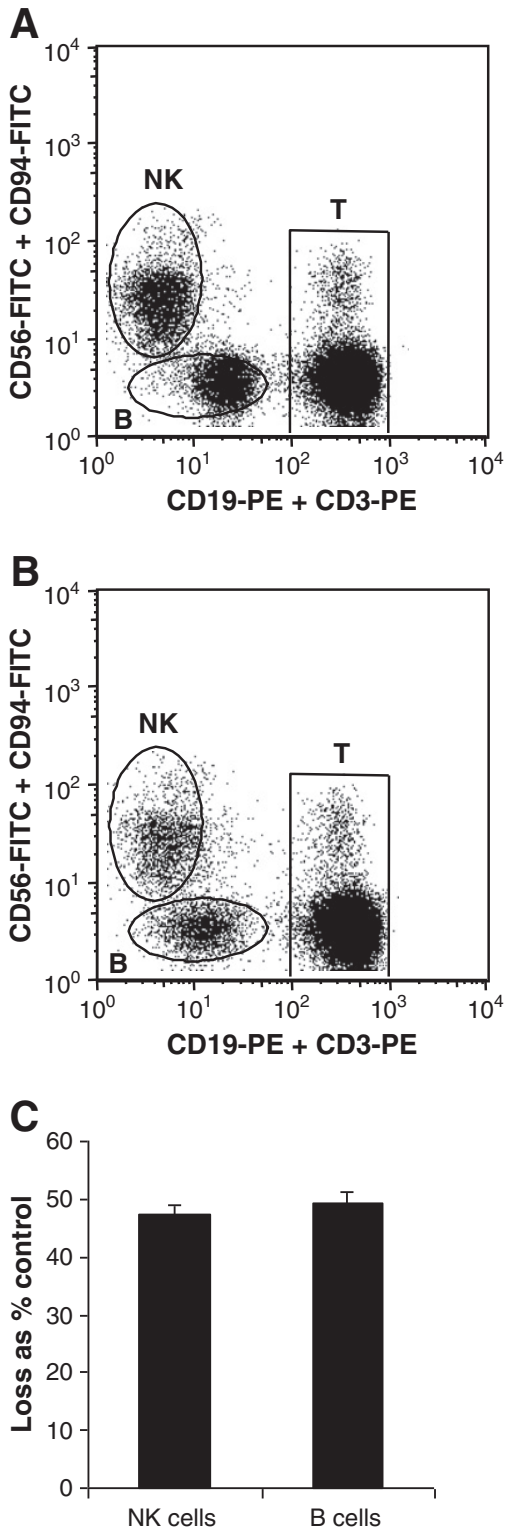

Fig. 6. Triplicate cultures were set up containing $5 \times 10^{4} \mathrm{PBMC}$ (control) alone (A) or with Rituximab (ADCC) (B). After $6 \mathrm{~h}$ cultures were analysed by immunofluorescence staining and flow cytometry. NK cells were defined as $\mathrm{CD}^{-}{ }^{-} \mathrm{CD} 56^{+} \mathrm{CD}^{+} 4^{+}$(15.7\%). B cells (16.8\%) and T cells (65.3\%) were identified with CD19-PE and CD3-PE. NK cells and B cells were quantified relative to T cells as an internal control (C). The $100 \%$ control values corresponded to $0.2524 \pm$ 0.0024 (NK/T ratio) and $0.2835 \pm 0.0068$ (B/T ratio).

\subsection{Autologous B cells are targets for ADCC and this is associated} with NK cell loss

The data in Fig. 6 show that NK cell mediated ADCC can be measured directly in PBMC without added target cells by the Rituximab-dependent killing of autologous B cells. The results are from one of 4 experiments with different donors. PBMC were cultured alone and with Rituximab. B cells and T cells were analysed using CD19-PE and CD3-PE mAbs, respectively, that were used at concentrations enabling a clear separation of the populations. NK cells were analysed using a combination of CD56-FITC and CD94-FITC. Gating of NK cells, B cells and $T$ cells is shown for control cultures and cultures containing Rituximab (ADCC). Interestingly the level of expression of CD19 is lower on B cells remaining in cultures containing Rituximab (Fig. 6A compared with Fig. 6B). NK cells and $\mathrm{B}$ cells were quantified using $\mathrm{T}$ cells as an internal control by using the ratio of $\mathrm{NK} / \mathrm{T}$ and $\mathrm{B} / \mathrm{T}$ in the cultures, respectively. The bar graph shows that both $B$ cells and NK cells are lost in cultures with Rituximab (Fig. 6C).

\section{Discussion}

The data presented in this paper show that target-induced NK loss (abbreviated as TINKL) provides a measure of NK cell responses to target cells, with respect to both natural killing and ADCC.

Target-induced NK loss refers to the loss of NK cells from within the lymphocyte gate as analysed by flow cytometry. The lost NK cells would be bound to target cells, and this can lead to activation-induced NK cell death as shown by others (Jewett and Bonavida, 1996). Some NK cells remaining within the lymphocyte gate bound Annexin- $\mathrm{V}$, an early marker of apoptosis, indicating that these NK cells have interacted with and then disengaged from target cells. That only a proportion of NK cells are lost and/or are programmed for apoptosis following target cell interaction is consistent with the knowledge that NK cells can kill multiple target cells (Bhat and Watzl, 2007), and that depending on the target cells NK cells are stimulated to proliferate (Warren, 1984; Warren et al., 1995; Warren and Kinnear, 1999).

The fact that a substantial proportion of NK cells are lost from analysis in the lymphocyte gate following culture with target cells should be considered when interpreting the CD107a assay to quantify NK cell responses. It is on the remaining NK cells present in the lymphocyte gate that CD107a expression is measured. These NK cells presumably have had a weaker interaction with target cells and have become disengaged from them. Thus measuring CD107a expression on those NK cells could underestimate NK cell responses.

Measurement of NK cell responses to tumour cells by quantifying TINKL could readily be accomplished in routine clinical testing. The assay set up is convenient since PBMC can be isolated the previous day or thawed from liquid nitrogen storage, and then cultured overnight prior to the incubation with target cells. The assay is very robust, and although in this study all assays were done in triplicate, single determinations would be adequate for routine analysis of NK cell responses. NK cells can be quantified using $\mathrm{T}$ cells as an internal reference without the need to add counting beads. ADCC can be measured without added target cells by monitoring the loss of autologous B cells in cultures of PBMC and the CD20 mAb Rituximab. TINKL could have application in assessing the efficacy of Rituximab prior to its use in the treatment of lymphoma patients.

It is likely that evaluation of NK cell responses will become routine in the clinical laboratory, not only in evaluating responses to tumour cells, but in establishing vaccine efficacy particularly where there is a demonstrated protective role for NK cells against a particular infection (Horowitz et al., 2010a; Horowitz et al., 
2010b). Vaccination enhancing T cell immune responses would lead to IL-2 production which would be expected to promote NK cell expansion (Warren, 1996) and enhance NK cell responses. In these settings TINKL should prove a useful and cost effective method for evaluating NK cell responses.

\section{Acknowledgements}

This work was funded by grants from the ACT Health and Medical Research Support Program and The Canberra Hospital Private Practice Fund. The author wishes to thank Dr Dipti Talaulikar for providing Rituximab, Ms Yvonne Gonzales-Cendales for technical help in some of the experiments, Dr Ben Quah for help with the FlowJo analysis, and Dr Matthew Cook, Dr Ben Quah and Prof Chris Parish for their helpful comments on the manuscript.

\section{References}

Alter, G., Malenfant, J.M., Altfeld, M., 2004. CD107a as a functional marker for the identification of natural killer cell activity. J. Immunol. Methods 294, 15-22.

Bhat, R., Watzl, C., 2007. Serial killing of tumor cells by human natural killer cells - enhancement by therapeutic antibodies. PLoS One 2, e326.

Brunner, K.T., Mauel, J., Cerottini, J.C., Chapuis, B., 1968. Quantitative assay of the lytic action of immune lymphoid cells on 51-Cr-labelled allogeneic target cells in vitro; inhibition by isoantibody and by drugs. Immunology 14, 181-196.
Cooper, M.A., Fehniger, T.A., Caligiuri, M.A., 2001. The biology of human natural killer-cell subsets. Trends Immunol. 22, 633-640.

Fauriat, C., Andersson, S., Bjorklund, A.T., Carlsten, M., Schaffer, M., Bjorkstrom, N.K., Baumann, B.C., Michaelsson, J., Ljunggren, H.G., Malmberg, K.J., 2008. Estimation of the size of the alloreactive NK cell repertoire: studies in individuals homozygous for the group A KIR haplotype. J. Immunol. 181, 6010-6019.

Horowitz, A., Behrens, R.H., Okell, L., Fooks, A.R., Riley, E.M., 2010a. NK cells as effectors of acquired immune responses: effector $\mathrm{CD} 4{ }^{+} \mathrm{T}$ cell-dependent activation of NK cells following vaccination. J. Immunol. 185, 2808-2818.

Horowitz, A., Newman, K.C., Evans, J.H., Korbel, D.S., Davis, D.M., Riley, E.M., 2010b. Cross-talk between T cells and NK cells generates rapid effector responses to Plasmodium falciparum-infected erythrocytes. J. Immunol. 184, 6043-6052.

Jewett, A., Bonavida, B., 1996. Target-induced inactivation and cell death by apoptosis in a subset of human NK cells. J. Immunol. 156, 907-915.

Warren, H.S., 1984. Differentiation of NK-like cells from OKT3-, OKT11 ${ }^{+}$, and $\mathrm{OKM} 1^{+}$small resting lymphocytes by culture with autologous $\mathrm{T}$ cell blasts and lymphokine. J. Immunol. 132, 2888-2894.

Warren, H.S., 1996. NK cell proliferation and inflammation. Immunol. Cell Biol. 74, 473-480.

Warren, H.S., Kinnear, B.F., 1999. Quantitative analysis of the effect of CD16 ligation on human NK cell proliferation. J. Immunol. 162, 735-742.

Warren, H.S., Kinnear, B.F., Phillips, J.H., Lanier, L.L., 1995. Production of IL-5 by human NK cells and regulation of IL- 5 secretion by IL-4, IL-10, and IL12. J. Immunol. 154, 5144-5152.

Yamauchi, A., Taga, K., Mostowski, H.S., Bloom, E.T., 1996. Target cell-induced apoptosis of interleukin-2-activated human natural killer cells: roles of cell surface molecules and intracellular events. Blood 87, 5127-5135. 